\title{
Detección de antígenos virales en lactantes con infección respiratoria aguda baja
}

\author{
M. Lina Boza C.' ; Waldo Castillo M. ${ }^{1}$; Luisa Cancino D. ${ }^{2}$ \\ Miriam Muñoz $\mathrm{O}^{2} ;$ Gregorio Aguilera $\mathrm{A}^{3}$
Viral antigens in nasopharingeal exudates from infants with acute lower respiratory tract infections

\begin{abstract}
To measure the frequency and distribution of positive identification of viral antigens by indirect immunofluo. rescence (IF) in patients aged 24 months or less with lower respiratory tract infections. IF reactions for respiratory syncytial virus (RSV), adenovirus (AQV), parainfluenza 1, 2 and 3 vi, us (PIV), influenza a and b (IVa and IVb) were carried on nasopharyngeal secretions from 660 children admitted before 24 months of age to the pediatric wards of a metropolitan general hospital at Santiago. Chile, because of clinical and radiological evidence of severe lower respiratory tract infection (pneumonia, pneumonitis, bronchiolitis, apnea plus lung infiltrates). Positive IF were obtained from 322 cases $(48.8 \%$ ). Most frequenkly identified antigens were those of RSV (56.2\%) y ADV (26.7\%). Lethality among palients with positive IF was $9.9 \%$ and $6.2 \%$ in those with negative IF ( $p$ : NS), but in 24 of 32 (75\%) positive If patients that died, ADV was the isolated antigen, the risk of death being in these cases significantly higher than in patients with other antigens or negative $|F| Z$ crit -1.65 vs. $Z$ calc $-1,178 \mid$. Apnea was the main clinical sympton in 46 patients. all under 3 months of age. 19 of them had posltive IF reactions that with only one exception corresponoed to RSW.
\end{abstract}

iKey words: jower respiratory infections, pneumonia, pneumonitis, bronchiolits, apnea, viral antigens, immuno. fluorescence, adenovirus, respiratory synevtial virus.t

Las infecciones virales del árbol respiratorio pueden ser asintomáticas o causar molestias que varían de mínimas a severas, pudiendo amenazar la vida o producir secuelas como bronquiolitis obliterante ${ }^{1}$, bronquiectasias ${ }^{2}$, pulmón hiperlúcj$\mathrm{do}^{3,4}$, e hiperreactividad bronquial recurren. te $\mathrm{e}^{\mathrm{s}-\mathrm{T}}$, todas las cuales pueden, a su vez, causar hipertensión pulmonar y corazón pulmonar crónico.

Aun cuando los exámenes microbiológicos corrientes tienen bajo rendimiento en la detección de bacterias causantes de infecciones respiratorias agudas bajas (IRAB) en lactantes, la evidencia sugiere que, en países desarrollados y

1. Servicio de Pediatría, Unidades de Broncopulmona. res e Ingreso, Hospital San Borja Arriarán, SSMC y Departamento de Pediatria, Campus Centro, Facultad de Medicina de la Universidad de Chile.

2. Kinesiólogos, Servicio de Medicina Física y Rehabjlitación, Hospital San Borja Arriarán.

3. Tecnólogo Médico, Unidad de Inmunología, Laboratorio Central, Hospit al San Borja Arriarán. el nuestro, la mayoria de elias es causada por virus. La identificación de agentes virales mediante estudios serológicos y cultivos tiene, en los casos aludidos, inconvenientes de coste y tardanza en la obtención de resultados, que los hacen poco prácticos para propósitos clínicos. Por otra parte, la pesquisa de antigenos virales en secreciones respiratorias, si bien no establece necesariamente relaciones de causalidad, ofrece información valiosa en la presunción etiológica, para orientar el tratamiento en los primeros días después del ingreso, especialmente cuando los demás elementos de juicio no sugieren otros tipos de agentes infecciosos ${ }^{8,9}$. En nuestro hospital no se disponía de datos suficientes sobre la prevalencia y distribución de dichos antígenos virales en niños menores de 2 años con IRAB, por lo que en 1988 se inició una investigación operativa, cuyos resultados se exponen a continuación, con el propósito de describir la frecuencia con que algunos de ellos se detectan, mediante inmunofluorescencia indirecta (IF), en ese tipo de pacientes. 


\section{Pacientes y Métodos}

Dyrante los meses de abril a septiembre de los años 1988, 1989 y 1990, con lá única limitante de la disponibilidad de reactivos para detección de ant ígenos virales por inmunofluorescencia, se estudió la mayor cantidad posible de niños de 0 a 24 meses de edad ingresados al Hospital San (Francisco de) Borja-(Manuel) Arriarár de Santiago (HSBA) por IRAB grave, definida como una enfermedad inflamatoria, febril, de la vía respiratoria -desde la laringe a los akéolos pulnonaresacompañada o no de síndrome bronquial obstructivo, con evidencia radiológica de compromiso del intersticio o el parénquima pulmonares o ambos, con o sin signos clínicos o radiológicos de híperinsuflación pulmomar. La enfermedad se consideró grave si el paciente cumplía al menos con tres de los siguientes ctitetios: temperatura mantenida sobre $38,5^{\circ} \mathrm{C}$ rectal; puntuación de Tal ${ }^{10}$ igual o mayor que 8: signos radiologicos de hiperinsuflación o compromiso intersticial diưso, con o sin signos de relleno alveolar o atelectasia, en la radiografía de tórax; hipoxomia arterial con o sin hipercapnea; episodios de aphea con bradicardia, de 20 o más segundos de duración. Sólo se incluyeron pacientes previa. mente sanos, cuya enfermedad actual tuviese una duración inferior a 5 dias. A todos cllos se les tomaron muestras de aspirado nasofaríngeo, para reacciones de inmunofluorescencia a antígenos de virus sincicial respiratorí (VSR), adenovirus ( $A D V$ ), virus parainfluerza 1,2 y 3 (VPI), virus influenza a y b (VIa y VIb). Las muestras fueron obtenidas por los kinesiólogos participantes, a más tardar al séptimo día de evolución con la técnica descrita por Méndez ${ }^{1}$. Los frotis fueron procesados en el laboratorio del HSBA y leídos en ej Instituto de Salud Pública de Chile, en el año 1988; posteriormente todo el procedimiento se realizó en el HSBA.

Los pacientes recibieron oxígeno según sus necesidades $v$, en caso de sindrome bronquial obstructivo, salbutamol o fenoterol en nebulizaciones o aerosol, con o sin bromuro de ipratropio. Los esteroides se emplearon sólo en casos de síndrome obstructivo sovero o progresivo. Los antibióticos se usaron en los enfermos con Iti negativa que, además, tuviesen en la sangre profeina C reactiva $\Rightarrow 40 \mathrm{mg} / \mathrm{dl}$, velocidad de sedincntación globular $>40 \mathrm{~mm} / \mathrm{h}$ o más de 15000 leucocitos por $\mathrm{mm}^{3}$, por separado o en asociación, y en paciente con IF positiva con manifestaciones clínjeas y radiológicas de agravamiento de su evolución y aparición posteriou de los índices recién descritos.

De esta manera se estudjaron 660 nuestras de igual numero de pacientes, se analizó la frecuencia absoluta y relativa de las jdentificaciones positivas $y$ se hicieron comparaciones de edad, sindrome chínico y letalidad entre lactantes afectados por IR AB con reacciones de IF positivas y negativas. Para el análjsis estadistico de los resultados se emplé la prueba $t$ de Student, asignando significación a valores de $\mathrm{p} \leqslant 0.05$.

\section{Resultados}

En $322(48,8 \%)$ pacientes se obtuvieron resultados positivos con $\mathrm{IF}, 188(58,4 \%)$ eran varones. Entre los niños con IF negativa, 202 $(59,7 \%)$ eran de sexo masculino. La edad entre pacientes con IF positivas y regativas fue, respectivamente: $<6$ meses en $202(62,7 \%)$ vs. $221(65,3 \%) ; 6$ a 12 meses en $78(24,2 \%)$ vs. $81(24,05 \%)$ y $>12$ meses en $42(13,1 \%)$ y 36 $(10,7 \%)$, sin diferencias significativas. Las manifestaciones clinicas de la IRAB se agruparon, de acuerdo con las definiciones del metodo, en síndrome bronquial obstructivo (SBO), bronconeumonía (BN), combinaciones de $\mathrm{SBO}$ con $B N$, y apnea, predominando claramente la combinación aludida sobre las restantes. Cabe destacar que los casos de apnea sólo ocurrieron en niños de 3 meses o menos de edad (tabla 1).

Los antígenos virales detectados con mayor frecuencia fueron, en orden decreciente: los de

Tabla 1

Distribución por edad de 660 lactantes con infecciones respiratorias agudas bajas e inmunofluorescencia positiva (n: 322 j o negativa (n: 338) para ant ígenos virales, según edad y diagnóstjco clínico

\begin{tabular}{|c|c|c|c|c|c|c|c|c|c|c|c|c|c|c|c|}
\hline & \multicolumn{3}{|c|}{$\mathrm{SBO}$ con $\mathrm{BN}$} & \multicolumn{4}{|c|}{ SBO } & \multicolumn{4}{|c|}{ BN } & \multicolumn{4}{|c|}{ Apnea } \\
\hline & IF+ & \multicolumn{2}{|c|}{ IF+ } & \multicolumn{2}{|r|}{$\mathrm{IF}+$} & \multicolumn{2}{|c|}{$\mathrm{IF}-$} & \multicolumn{2}{|r|}{ IF+ } & \multicolumn{2}{|c|}{ IF - } & \multicolumn{2}{|c|}{$\mathrm{IF}+$} & \multicolumn{2}{|c|}{ IF- } \\
\hline & n $\%$ & n & $\%$ & $n$ & $\%$ & n & 4 & $\mathbf{n}$ & $\%$ & $\mathrm{n}$ & $\%$ & $\mathrm{n}$ & $\%$ & $\mathrm{n}$ & $\%$ \\
\hline$<6$ meses 99 & $9(30,7)$ & 119 & $(30,7)$ & 57 & $(17,5)$ & 38 & $(11,9)$ & 36 & $(11, \mathbf{1})$ & 43 & $(12,2)$ & 19 & $(5,9)$ & 27 & $(8,0)$ \\
\hline 6.12 meses 38 & $8(11,9)$ & 23 & $(6,7)$ & 25 & $(7,6)$ & 30 & $(8,8)$ & 27 & $(8,5)$ & 27 & $(8,0)$ & - & - & 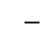 & - \\
\hline$>12$ meses 4 & $(1,2)$ & 7 & $(2,0)$ & 10 & $(3,0)$ & 12 & $(, 37)$ & 7 & $(2,2)$ & 12 & $(3,7)$ & - & - & - & - \\
\hline
\end{tabular}

BN: Neumonía, bronconeumonía o imágenes de condensación alveolar.

SBO: Síndrome bronquial obstructivo, clínico, ra diológico o ambos. 
$\operatorname{VSR}(56,2 \%)$ y ADV $(26,7 \%)$. En 32 casos, $10 \%$, hubo detecciones mixtas, siendo las asociaciones más frecuentes las de VSR con ADV ( $\mathrm{n}$ : 14), VIb (n: 12) y VPI (n: 4), con un caso cada una de VSR con VIa y ADV con VIb (tabla 2). Entre los 19 casos de lactantes menores con apnea e IF positiva los resultados de ésta demostraron casi siempre (95\%), con una sola excepción, antígeno de VRS.

De los 660 pacientes incluidos en el estudio, 53 fallecieron $(8,0 \%) ; 32$ tenían IF positiva $y$ representan a $9,9 \%$ de este grupo de 322 enfermos. La letalidad entre los 338 niños con IF negativa fue de 6,2\% ( $\mathrm{p}$ NS). Entre los 32 niños con IF positiva que murieron, en 24 (75\%) se identificaron antígenos para $\mathrm{ADV}$ (incluido un aisjamiento mixto con Vib), 5 tenían menos de 3 meses, 7 de 3 a 6 meses y 12 más de 6 meses de edad. Ел 8 (25\%) se habia aislado antigenos de VSR (incluida una identificación mixta con Vla), 4 de ellos tenían menos de 3 meses, 2 de 3 a 6 meses y 2 más de 6 meses de edad. La probabilidad de morir entre los pacientes de cualquier edad con antigenos ADV fue signifi. cativamente mayor al nivel establecido ( $Z$ crítico $\cdot 1,65$ vs. $Z$ calculado -- 1,178 ).

\section{Discusión}

Este trabajo resume la experiencia de tres años con la prevalencia de los antígenos virales elegidos, en una muestra de lactantes con infecciones respiratorias agudas bajas, seleccionados por su gravedad $y$ por un tiempo de evolución inferior a 1 semana. La mayor frecuencia de identificación de VSR en los pacientes que ingresan, como en los que presuntamente se infectan en el hospital, ha sido descrita previa-

\section{Tabla 2}

Trecuencia relativa de los ant ígenos virales detectados por inmunofluorescencia indirecta en 322 de 660 lactantes con infecciones respiratorias agudas bajas

\begin{tabular}{lrr}
\hline & $\mathbf{n}$ & $\%$ \\
\hline Virus sincicial resp. & 181 & 56,2 \\
Adenovirus & 86 & 26,7 \\
Virus parainfluenza & 14 & 4,3 \\
Virus influenż b & 9 & 2,8 \\
Asociación de virus* & 32 & 10,0 \\
\hline
\end{tabular}

* Ver distribución en el texto. mente ${ }^{12,13}$ y es coincidente con nuestros resultados, en los que el ADV ocupó el segundo lugar, con una frecuencia mayor que en otras series chilenas ${ }^{13-16}$, pero dentro del margen superior de las proporciones detectadas en otros parses, que es de 4 a $28 \% 14,15,17$, lo que en parte podria deberse a la selección de pacientes más graves $\mathrm{y}$, tal vez, a una incidencia real más ele. vada de las infecciones por este agente, cuya demostración excede las posibilidades metodológicas de este estudio.

La distribución de los antígenos virales identificados fue similar en ambos sexos. El ligero predominio de varones en la muestra, si bien no es estrictamente significativo, podría reflejar mayor incidencia de JRAB grave en este sexo. La aparentemente mayor susceptibilidad de los menores de 6 meses es posiblemente real y también derivada de la mayor frecuencia de pacientes más graves entre ellos, lo que es un hecho conocido ${ }^{9}$, relacionado con la falta de protección inmunitaria y las características anatomofuncionales del pulmón a su edad. La mayor letalidad asociada a IF positiva por adenovirus ha sido también reconocida previamente ${ }^{17}$. No estamos en con. diciones de descartar la posibilidad que, entre nuestros pacientes, puđiese haber ocurrido un serotipo de ADV particularmente agresivo, que no fue posible identificar por limitación de recursos. La asociación de infecciones por VSR $y$ apnea en menores de 3 meses merece ser destacada. La causa de la apnea no se conoce bien, aunque algunas hipótesis la relacionan con diferencias de respuesta al arco reflejo de la vía aé. rea superior, como consecuencia de la cual los estímulos locales producirian apnea obstructiva o mixta en lugar de $\operatorname{tos}^{28}$.

\section{Resumen}

Con el propósito de medir la frecuencia con que se aíslan determinados antígenos virales, en niños con infecciones respiratorias agudas bajas graves (IRAB), se realizaron ensayos de inmunofluorescencia (IF) para antígenos de virus sincicial respiratorio (VSR), adenovinus (ADV), virus parainfluenza 1,2 y 3 (VPI), virus influenza a y b (Vla y VIb) en las secreciones nasofarin. geas de 660 niños menores de 24 meses que cumplían con criterios clínicos y radiológicos de IRAB grave comúnmente aceptados. En 322 casos $(48,8 \%)$ se obtuvieron resultados positi- 
vos. Los antígenos más frecuentemente identificados fueron los de VSR $(56,2 \%)$ y $A D V$ $(26,7 \%)$. La letalidad en nifos con JRAB e IF positiva fue $9,9 \%$ y $6,2 \%$ en aquellos con IF negativa (p NS). En 24 de 32 (75\%) pacientes fallecidos con IF positivas, el antígeno identificado correspondia a ADV, siendo el resgo de morir en estos casos significativamente mayor que en el de pacientes portadores de otros antígenos o ninguno ( $Z$ crítico $-1,65$ vs. $Z$ calculado $-1,178)$. La apnea fue la principal manifestación clínica en 46 pacientes, todos menores de 3 meses y 19 con reacciones de inmunofluorescencia positivas que, con una sola excepción, correspondian a antigenos de VSR.

(Palabras claves: lnfección respiratoria baja, bronconeumonia, neumonia, neumonitis, bronquiolitis, apnea, antígenos virales, inmunofluorescencia, adenovirus, virus sincicial respira. torio.)

\section{Referencias}

1. Hardy $K$, Schidiow $D$, Zoeri N: Obliterative Bronchiolitis in children. Chest 1983; 3:460-466.

2. Simila $S$, Lanning $P$ : Chronic lung damage cau sed by adenovirus type 7; A ten year follow up study. Chest 1981; 80:127-131.

3. Daniel T, Wooddring $J$, Mac Vandeviere $H$, Witson $D$. Sawyer James syndrome. Unilateral hyperlucent lung. Clin Pediatr (Phila) 1984; 23: 393-397.

4. Wu $E$, Flores $M$, Jerez $E$ : Pulmón hiperlúcido uniJateral por adenovirus. Rev Chil Pediatr 1990; $61: 41.44$.

5. Mallory G, Motoyama E, Koumbourlis A, Mutich $R$, Nakayama $D$ : Bronchial reactivity in infants in acute respiratory faihure with viral bronchiolitis. Pediatr Pulmonol 1989; 6: 253.259.

5. Dutverman $E$, Neijend $H$, Von Strik $R$, Affourtt $M$, Herrenbijn $\boldsymbol{X}$ : Lung function and bronchia! responsiveness in children who had infantile bronciolitis. Pediatr Pulmonol 1987; 3: 38-44.
7. Haltan M, Keens $T$, Lapierre JG, Levison $H$ : Pulmonary function abnormalities in sympton free children after bronchiolitis. Pediatrics 1977; 59 : 683-688.

8. Méndez $B$, Goldenberg $E$. Vicente $M$, Diaz $A$, Farios $p$ : Infecciones respiratorias agudas bajas por virus sincicial respiratorio. Diagnóstico rápido por técnica de inmunofluorescencia, Rev Chil Pediatr 1987; 30:235-240.

9. Centi E, Díaz A, Vicente M, Escobar AM, Mar inez $F$. Pinto $R$ : Etiología de las infecciones respiratorias bajas agudas en lactantes hospitalizados. Rev Chil Pediatr 1991;62: 155-166.

10. Tal A, Bavilski $C$, Yohai $D$, Bearman JE, Gordischer $R$. Moses $S w$ : Dexamethasone and salbutamol in the treatment of acute wheezing in infants. Pediatrics 1983; 71: 13-18.

11. Brandt D, Hyun WK, Ariobio J, Jeffnes B, Wood $S C$. Chanock $R$ : Epidemiology of respiratory sincitial virus infection in Washington D.C. Am J Epidemiol 1973; 98: 355-365.

12. Burdach $R$, Vicente $M$, Carrasco $L$, Sepúlveda $H$, Aguilera $G$, González $Q$ : Etiología viral en atecciones broncopulmonares agudas y recidivantes de la infancia. Revista Chilena de Infectología 1987; 4: 104-109.

13. Van der Veen $J$ : The role of adenovirus in respiratory disease. Am Rev Resp Dis 1963; 88: 167180.

14. Edwards $K M$, Thomson S, Paolini J: Adenovirus infections in young children. Pediatrics 1985; 76: $420-424$.

15. Almarza $M I$, Wu E, Vicente $M$. Torres $G$, Garay B. Alvarez $A M$ : Infecciones respiratorias agudas intrahospitalarias. Rev Chil Pediatr 1990; 61: 185-188.

16. Wade UG: Adenoviruses. Principles and Practice on Clinical Virology. Ed Zuckerman AJ, Wiley J and Sons Ltd, London 1987.

17. Wu $E$, Martinez $V$, Alvarez AM, Larrañaga $G$, $V$ Véo $H$ : Casos fatales de infección por adenovirus. Rev Chil Pediatr 1990; 61: 177.184.

18. Pickens $D$. Schefft $G$, Storch $G$, Thach B: Cha racterization of prolonged apnejc episodes associated with respiratory syncytial virus infections. Pediatr Pulmonol 1989; 6: 195-201. 